\title{
Effect of Level of Sensitization on Gait in Chronic Low Back Pain: Insights from a Machine Learning Approach
}

\section{Xiaoping Zheng ( $\nabla$ x.zheng@umcg.nl )}

University of Groningen Faculty of Medical Sciences: Universiteit Groningen Faculteit Medische Wetenschappen https://orcid.org/0000-0002-7556-130X

\section{Michiel Reneman}

University of Groningen: Rijksuniversiteit Groningen

Jone Ansuategui Echeita

University of Groningen: Rijksuniversiteit Groningen

\section{HR Schiphorst Preuper}

University of Groningen: Rijksuniversiteit Groningen

Herbert Kruitbosch

University of Groningen: Rijksuniversiteit Groningen

\section{Egbert Otten}

University of Groningen: Rijksuniversiteit Groningen

\section{Claudine Lamoth}

University of Groningen: Rijksuniversiteit Groningen

\section{Research}

Keywords: Low back pain, Central sensitization, Supervised machine learning, Gait, Daily life

Posted Date: May 6th, 2021

DOI: https://doi.org/10.21203/rs.3.rs-495586/v1

License: (c) (1) This work is licensed under a Creative Commons Attribution 4.0 International License. Read Full License 


\section{Effect of level of Sensitization on gait in Chronic low back}

\section{pain: insights from a machine learning approach}

3 Xiaoping Zheng ${ }^{*}$, Michiel Reneman ${ }^{2}$, Jone Ansuategui Echeita ${ }^{2}$, HR Schiphorst

$$
\text { Preuper }^{2} \text {, Herbert Kruitbosch }{ }^{3}, \text { Egbert Otten }^{1}, \text { Claudine Lamoth }^{1}
$$

*Correspondence: $\underline{x . z h e n g @ u m c g . n 1}$

6 1. University of Groningen, University Medical Center Groningen, Department of Human Movement Sciences, Groningen, Netherlands

8 2. University of Groningen, University Medical Center Groningen, Center for Rehabilitation,

$9 \quad$ Groningen, the Netherlands

10 3. University of Groningen, Center for Information Technology, Data science team, Groningen,

11 the Netherlands 


\section{Abstract}

13 Background: Despite central sensitization (CS) often presents in patients with chronic

14 low back pain (CLBP), there is a lack of quantitative and qualitative analysis of the 15 effect of CS on gait performance. The study aimed to investigate the daily-living gait 16 performance of patients with CLBP with low and high CS levels (CLBP- and CLBP+, 17 respectively).

18 Method: Forty-two patients with CLBP were included. CS was assessed by Central 19 Sensitization Inventory (CSI). Patients were classified according to low or high CSI 20 score (23 CLBP- and 19 CLBP+). Patients wore a 3D accelerometer for about one week.

21 From each patient, 4 days of accelerometer-data were randomly selected. For each day 22 data, continuous gait cycles were extracted by using a Fast Fourier Transform-based 23 and a zero-cross method. For all gait cycles in one day, 36 gait outcomes representing 24 variables related to pace, regularity, smoothness, local stability and predictability of 25 gait were calculated. A Random Forest classifier was trained to classify CLBP- and CLBP+ groups based on gait outcomes and SHapley Additive exPlanations (SHAP) method was used to explain the differences between groups in gait outcomes.

Results : The Random Forest classifier could accurately recognize the CLBP- and

29 CLBP + groups $($ accuracy $=84.4 \%$, F1-score $=82.6 \%$ ). SHAP reported that the most 30 differences between CLBP- and CLBP+ groups were: index of harmonicity-vertical 31 and harmonic ratio-mediolateral (gait smoothness), stride frequency variabilitymediolateral/anteroposterior, stride length variability (gait variability), stride

33 regularity-mediolateral (gait regularity), maximal Lyapunov exponent- 
34 vertical/mediolateral and maximal Lyapunov exponent per stride-vertical (gait 35 stability), and sample entropy-anteroposterior (gait predictability).

36 Conclusions: CLBP- and CLBP+ presented different motor control strategies. CLBP-

37 presented a more "loose" control, including higher gait smoothness and stability.

38 CLBP+ presented a more "tight" control, including a more regular, less variable and 39 more predictable gait pattern.

40

41 Keywords: Low back pain, Central sensitization, Supervised machine

42 learning, Gait, Daily life. 
Chronic low back pain (CLBP) is one of the most prevalent chronic musculoskeletal pains in the world [1]. It is responsible for high treatment costs, sick leave and individual suffering and it represents a significant socioeconomic burden [2]. For 85\% to $90 \%$ of patients with CLBP, the relation between pathoanatomical and clinical presentations is weak [3] and, therefore, it is classified as nonspecific CLBP [4]. In CLBP, and other chronic musculoskeletal disorders, central sensitization (CS) might be present [5]. CS is defined as "increased responsiveness of nociceptive neurons in the central nervous system to their normal or subthreshold afferent input" [6] and manifests as mechanical hypersensitivity, allodynia and hyperalgesia [7]. A considerable number of people need treatment for CLBP. Although the overall efficacy of CLBP rehabilitation programs is positive, the effect sizes are modest [8].

Correctly recognizing the physical and psychosocial factors perpetuating pain and physical disability of patients with CLBP remains a challenge [9]. Altered motor control of patients with CLBP could possibly contribute to the persistence of CLBP [10]. Altered motor control could affect daily-living activities, as patients with CLBP often exhibit altered movement patterns and motor control strategies; probably in order to avoid painful movement, such as walking [11]. Walking is one of the abilities most

62 affected by CLBP. Many clinicians may intuitively identify "abnormal" gait patterns in 63 patients with CLBP, but identification and objectifying of specific "abnormal" gait 64 features is challenging. During walking, it is suggested that patients often adopt a 

"protective guarding" or "splinting" strategy [12] to avoid painful movements of the

66 spine. These adaptations may lead to a slower and less flexible gait pattern [13].

67 Evidence for this, however, is not ubugious. Studies between patients with CLBP and 68 healthy controls, observed inconsistent evidence regarding preferred walking velocity 69 [13] [16], stride length [14] [17], and stride-to-stride variability [15] [18].

71 A possible explanation for these inconsistencies might be an unknown heterogeneity 72 within the samples, such as the presence of CS. CS could plausibly be related to the 73 inconsistent results, because the presence of high CS levels is associated with long74 lasting chronic pain [19] and movement may be changed due to pain. Also, general gait 75 features such as walking speed and stride length, might not be sensitive enough to detect small differences between patients with high and low levels of CS. In addition to stride related parameters, gait outcomes that reflect gait quality in terms of regularity, 78 synchronization, smoothness, local stability and predictability, are sensitive to detect 79 differences in gait performance. These gait outcomes were successfully used to detect 80 the differences between age groups [20], older adults with and without fall risk [21], 81 and patients with and without Parkinson's disease [22]. Despite the fact that the effects 82 of CLBP on gait have been frequently investigated in controlled laboratory studies, 83 there are no studies about the relationship between CS levels and gait performance 84 under daily-living environment circumstances. 
Advances in wearable technology and machine learning methods offer new opportunities in gait data collection and analysis. Wearable technology allows researchers to record patients' physical activities in unobserved, daily-living environments over extended periods of time. This data can reflect the real gait performance of the patients since the controlled laboratory environment, while being

91 observed, may change the performance of patients [23]. The successful employment of

92 machine learning methods in gait analysis makes it possible to extract the most 93 informative gait outcomes from the accelerometer sensor data [20]. If patients with 94 lower and higher levels of CS walk differently, the machine learning methods will be

95 able to successfully recognize these differences by their gait outcomes. Many gait outcomes are not independent and interact with each other, such as gait speed and step regularity. Machine learning methods such as Random Forest approach, are able to process high dimensional and non-linear data structures and take the interrelation and interaction of the gait outcomes into consideration [20].

101 Therefore, the aim of this study was to analyze whether and how the presence of CS is related to differences in gait performance of patients with CLBP during daily life by

103 using a machine learning method. It was hypothesized that patients with CLBP and

104 higher levels of CS show differences in daily life gait performance, compared with 105 those with lower levels. 


\section{Patients}

110 This study included patients with primary CLBP who were recruited from the outpatient

111 Pain Rehabilitation Department of the Center for Rehabilitation of the University

112 Medical Center Groningen (CvR-UMCG). Primary CLBP is defined as low back pain

113 persistent for more than three months, with pain not being the result of any other

114 diagnosis. The patients were selected according to the following inclusion criteria: (a)

115 age between 18 and 65 years old at the time of recruitment; (b) admitted to the

116 interdisciplinary pain rehabilitation program; (c) could follow instructions; (d) signed

117 informed consent. Additionally, patients were excluded if they: (a) had a specific

118 diagnosis that would better account for the symptoms (e.g. cancer, inflammatory

119 diseases and/or spinal fractures); (b) had neuralgia and/or radicular pain in the legs; (c)

120 were pregnant.

122 The study was approved by the Medical Research Ethics Committee of the University

123 Medical Center Groningen (METc 2016/702) and conducted according to the principles

124 expressed in the Declaration of Helsinki. The data used in this paper was derived from

125 a larger study, of which protocol details were described elsewhere [19].

127 Data collection

128 Demographics were collected and standard clinical test were applied as part of the usual

129 care of CLBP patients that are referred to the outpatient Pain Rehabilitation Department 
130 of the Center for Rehabilitation. Assessments included: Visual Analogue Scale for pain

131 intensity (VAS Pain; 0-10), the Dictionary of Occupational Titles (DOT, the Pain

132 Disability Index (PDI; 0-70), the physical functioning subscale of the Rand36

133 questionnaire (Rand36-PF; 0-100), the Pain Catastrophizing Scale (PCS, 0-52), the

134 Injustice Experience Questionnaire (IEQ, 0-48), and the Brief Symptom Inventory (BSI

135 global severity index t-score (GSIT))(see Table 3).

136

137 Central sensitization (CS). The presence of CS-related manifestations was assessed

138 with section A of the Central Sensitization Inventory (CSI) [24]. Section A has 25-

139 items to assess the presence of common CS-related symptoms. Scores can range from

$140 \quad 0-100$ where a higher scoring represents a higher level of CS. A score lower 40 indicates

141 low CS level (CLBP-group) and a score of 40-100 is interpreted as high CS level

142 (CLBP+ group) [25].

143

144 Mann-Whitney U test was used to statistically test the differences between CLBP-

145 and CLBP+ groups for demographics and CSI scores.

147 Accelerometer data. The accelerometer data was collected between 2017 and 2019.

148 Patients were instructed to wear a tri-axial accelerometer (ActiGraph GT3X, Actigraph

149 Corporation, Pensacola, FL) at all times for about one week, excluding sleeping or

150 bathing times. The accelerometer was worn at the front right hip of the patient (at the

151 anterior superior iliac spine). Assuming a standing and upright position, the Y-axis 
152 pointed to the ground (vertical direction, V), Z-axis faced the walking direction

153 (anteroposterior direction, AP), and the X-axis was perpendicular to the walking

154 direction, pointing from a patient's right to left (mediolateral direction, ML). The

155 sampling frequency of the accelerometer was set to $100 \mathrm{~Hz}$ and the dynamic range was

$156 \pm 6$ gravity.

157

158 Data processing and analysis

159 Raw data segmentation

160 Each patient's accelerometer data was segmented into 24 hours span data segments

161 (from 12:00 P.M. to next day 11:59 A.M) to represent the activities during the days.

162 Data which was not completely covering this 24-hour span was discarded from the

163 analysis. Due to technical or personal reasons of the patient, not all patients were

164 collected a full week of data. In order to compare the data between different patients

165 fairly, 4 segments (representing 4 days) of each patient were included. Therefore, 7

166 patients who had less than 4 segments, were excluded and from patients with more than

1674 segments, 4 segments were randomly sampled. Figure 1a graphically shows the

168 process of the raw data segmentation.

169 


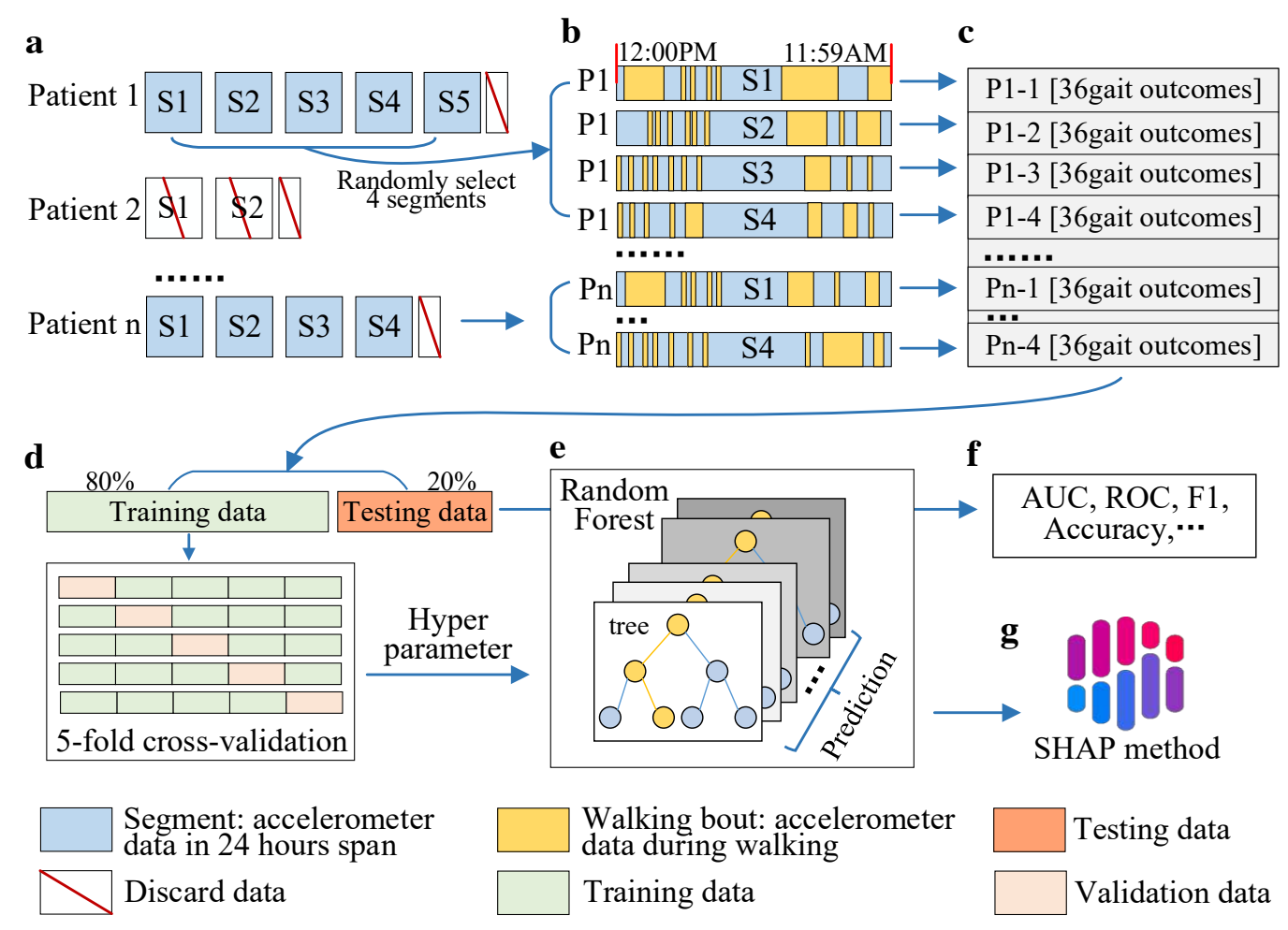

171 Figure 1. The data processing and analysis: (a) raw data segmentation, (b) walking

172 bouts extraction, (c) gait outcome vectors, (d) training and testing data preparation, (e)

173 Random Forest classifier, (f) accuracy evaluation, (g) feature importance.

\section{Walking bouts extraction}

176 The accelerometer data of the 4 segments was first smoothed by a low-pass filter with

177 a 2nd order Butterworth, filtered with a $20 \mathrm{~Hz}$ cut-off frequency, such that only

178 frequencies lower than $20 \mathrm{~Hz}$ remained. Subsequently, potential walking events were

179 detected by the Fast Fourier Transform (FFT) based method [26], which identified

180 periods with $0.5-3.0 \mathrm{~Hz}$ power spectrum values. To remove false walking events from

181 the potential walking periods, the zero-cross method [27] was employed. If the time

182 interval between any two adjacent walking events was shorter than 2 seconds, these

183 two walking events were merged into one walking bout. Finally, the walking bouts in 
each segment were extracted and their gait outcomes were calculated. Figure $1 \mathrm{~b}$

185 presents the walking bouts as the yellow vertical bars in the rectangle.

186

\section{Gait outcomes}

188 All walking bouts in one 24-hour segment were used to determine the total duration of

189 walking, the total number of steps, the maximum duration of a walking bout and the

190 maximum number of steps of a walking bout. And then, all walking bouts exceeding

19110 seconds were selected and cut into non-overlapping 10-second windows [28].

192 From the segment, each 10-second window was used to calculate different gait

193 outcomes, and these values were averaged over all 10-second windows in the segment

194 representing the patient's gait performance on that day.

196 The gait outcomes were divided into two categories, quantitative and qualitative gait

197 outcomes. From one segment, we obtained one gait outcome vector, including 36 gait

198 outcomes, based on the walking bouts (see Figure 1c). The detailed descriptions of the

199 quantitative and qualitative gait outcomes are presented in Table 1 and Table 2 -for 200 extended explanation of variables see reference [29]. 


\begin{tabular}{|c|c|c|}
\hline Catalog & Gait characteristic & Description and method \\
\hline \multirow{9}{*}{ Pace } & $\begin{array}{l}\text { Total duration of walking in } \\
\text { the day }\end{array}$ & $\begin{array}{l}\text { The accumulated time (in seconds) of the } \\
\text { walking bouts in one segment. }\end{array}$ \\
\hline & $\begin{array}{l}\text { Total number of steps in the } \\
\text { day }\end{array}$ & $\begin{array}{l}\text { The accumulated steps of walking bouts in one } \\
\text { segment. }\end{array}$ \\
\hline & $\begin{array}{l}\text { Maximum duration of a } \\
\text { walking bout }\end{array}$ & $\begin{array}{l}\text { Duration (in seconds) of longest walking bout } \\
\text { in one segment. }\end{array}$ \\
\hline & $\begin{array}{l}\text { Maximum number of steps of } \\
\text { a walking bout }\end{array}$ & $\begin{array}{l}\text { Maximum number of steps of one walking } \\
\text { bout in one segment. }\end{array}$ \\
\hline & $\begin{array}{l}\text { Walking speed (WS; mean, } \\
\text { variability) }\end{array}$ & $\begin{array}{l}W S=D / T \text {, where } D \text { is the distance (in } \\
\text { meters) and equals to the accumulated of step } \\
\text { length; } T \text { is the corresponding time (in } \\
\text { seconds). }\end{array}$ \\
\hline & $\begin{array}{l}\text { Stride length (SL; mean, } \\
\text { variability) }\end{array}$ & $\begin{array}{l}\text { SL }=2 \sqrt{2 l h-h^{2}} \text {, where } h \text { is the change in } \\
\text { height (in meters), } l \text { equals leg length (in } \\
\text { meters). } h \text { was calculated by a double } \\
\text { integration of the accelerometer signal in } \\
\text { vertical direction. SL is the sum of the adjacent } \\
\text { two step lengths. }\end{array}$ \\
\hline & $\begin{array}{l}\text { Stride time (ST; mean, } \\
\text { variability) }\end{array}$ & $\begin{array}{l}S T=n / f, \text { where } f \text { is the sample frequency } \\
\text { (in Hertz) and } n \text { is the number of }\end{array}$ \\
\hline & $\begin{array}{l}\text { Stride frequency (SF; mean, } \\
\text { variability-V/ML/AP) }\end{array}$ & $\begin{array}{l}\text { samples per dominant period derived from } \\
\text { autocorrelation. } \\
S F=f / n \text {. }\end{array}$ \\
\hline & $\begin{array}{l}\text { Root mean square of the } \\
\text { variability of the amplitude of } \\
\text { accelerations (RMS), }\end{array}$ & $\begin{array}{l}R M S=\sqrt[2]{\frac{1}{3}\left(x^{2}+y^{2}+z^{2}\right)} \text {, where } x, y, z \\
\text { represent the accelerometer signal (in meters } \\
\text { per second squared) in } \mathrm{x}, \mathrm{y}, \mathrm{z} \text { axis. }\end{array}$ \\
\hline
\end{tabular}

Table 2. Qualitative Gait Outcomes.

\begin{tabular}{lrr}
\hline Catalog & Gait characteristic & Description and method \\
\hline & SR is computed by using the unbiased
\end{tabular}
autocorrelation coefficient:

Stride regularity (SR; V, ML,

Regularity AP, All)

Gait symmetry index (GSI)

Index of harmonicity $(\mathrm{IH} ; \mathrm{V}$, Smoothness ML, AP, All)

Harmonic ratio (HR; V, ML, AP)
$A d(m)=\frac{1}{N-|m|} \sum_{i=1}^{N-|m|} A c c(i) \cdot A c c(i+m)$, where $\operatorname{Acc}(i)$ is the sample acceleration signal, $N$ the number of samples, and $m$ the number of time lag. The first peak of $A d(m)$ is $A d_{1}$ and it represents the stride regularity. Higher values (maximum 1.0) reflect repeatable patterns between strides.

GSI quantifies the ratio of the first and second peak of the $A d(m)$, as $A d_{1} / A d_{2}$. It is a measure of the degree of symmetry of the left and right lower limbs during walking.

$I H=\frac{P_{0}}{\sum_{i=0}^{6} P_{i}}$. It is the ratio of the power spectral density of the fundamental frequency $P_{0}$ and the sum of the power spectral density of the first six frequency $P_{i}$. IH quantifies gait smoothness., with higher values representing a smoother $(\max 1.0)$ gait pattern.

$H R=\frac{\sum P_{a}}{\sum P_{b}}$. In VT and AP directions, $\sum P_{a}=$ the sum of even power spectral and $\sum P_{b}=$ the sum of 
odd power spectral. In ML direction, $P_{a}$ is odd and $P_{b}$ is even. It reflects the rhythmicity of the walking patterns. Higher values mean more rhythmic

Sen $=-\ln (A / B)$, with $A=$

$d\left[A c c_{m+1}(i), A c c_{m+1}(j)\right]<r, \mathrm{~B}=$ $d\left[A c c_{m}(i), A c c_{m}(j)\right]<r . A c c_{m}(i)$ means the

Predictability Sample entropy (Sen; V, ML, AP) accelerometer signal vector from time $i$ to $m+$ $i-1 . d[]$ is the Chebyshev distance, and $r$ was set to 0.3 . Sen quantifies the predictability of a time series. Smaller values (minimum 0 ) indicate better synchronization between acceleration signals.

Maximal Lyapunov exponent max LyE, as calculated by the Rosenstein algorithm, (max LyE; V, ML, AP) quantifies the local stability of trunk acceleration patterns. The fitting window length was $60 / 100 *$

Stability

Maximal Lyapunov exponent $f$, where $f$ is the sample frequency, and the normalized per stride by time embedding dimension was set to 7 . Overall max (max LyE per stride; V, ML, LyE were calculated and normalized per stride by AP) time. Higher values represent greater sensitivity to local perturbations.

\section{Random Forest Classifier}

215 To separate CLBP- and CLBP+ groups by gait outcomes, a Random Forest classifier was used. The Random Forest classifier is considered as the optimal machine learning

217 classification method for the present data, because it performs well with (a) nonlinear and linear data; (b) high dimensional data, obsoleting dimensionality reduction; and (c) unbalanced and small data sets [30].

The input data of this method was $\langle S, L\rangle . S$ represents the gait outcome vectors of

222 patients and $L$ was its corresponding label. The definition of $S$ is: $S=$ $\left\{s_{1}, s_{2}, \ldots, s_{i}, \ldots, s_{m}\right\}$ and $s_{i}=\left[d_{1}, \ldots, d_{k}\right]$, where $s_{i}$ represents a gait outcome vector $i$ and $m$ is the number of all gait outcome vectors, $d$ represents a gait outcome and $k=36 . L=l_{1}, \ldots, l_{m}$, where $l \in\{C L B P-, C L B P+\}$. outcome vectors from $S$ and $n$ corresponding labels from $L$, with replacement. These 
231 outcomes from $s$. Therefore, $s_{i}^{\prime}=d_{1}^{\prime}, \ldots, d_{j}^{\prime}$ and $S_{b}^{\prime}=s_{1}^{\prime}, \ldots, s_{n}^{\prime}$. Step three:

232 Training a decision tree $f_{b}$ on $S_{b}^{\prime}, L_{b}$. Step four: Repeat steps one to three 1000 times 233 and combine the decision trees into an ensemble, called random forest, that predicts by 234 voting (see Figure 2).

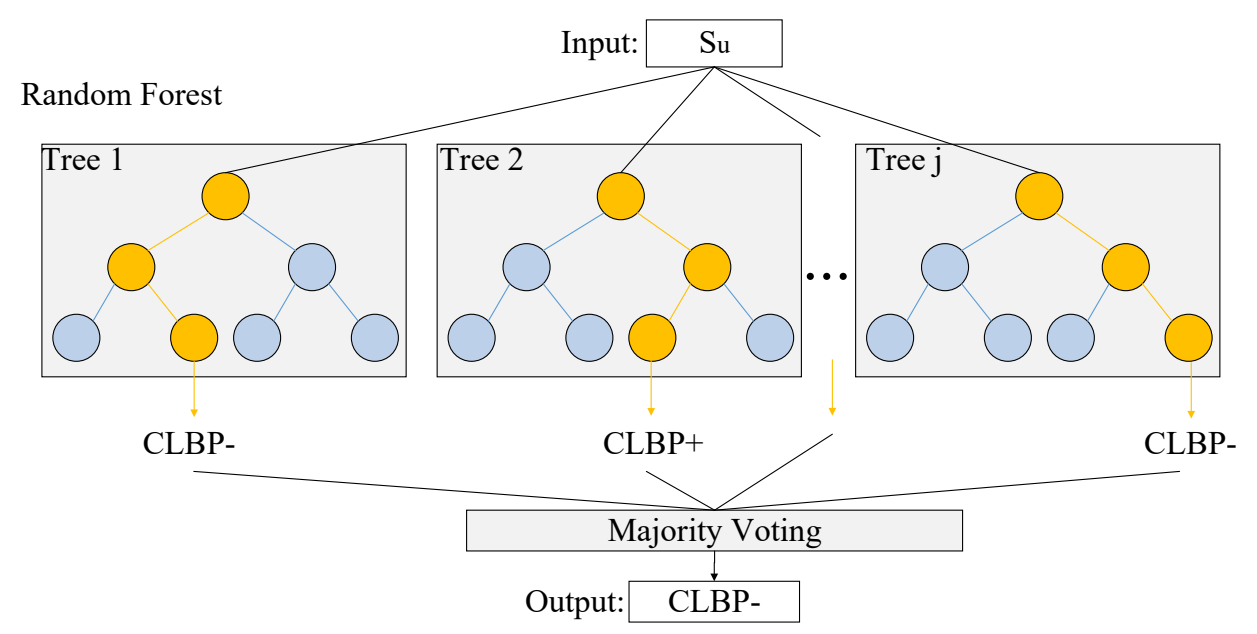

Figure 2. Architecture of random forest.

Before training the Random Forest classifier, $80 \%$ of patients were randomly selected and their corresponding gait outcome vectors were used as the training data. The gait outcome vectors of the remaining $20 \%$ of patients were used as the testing data. In order to avoid overfitting of the hyperparameters, a 5-fold cross-validation method was used to estimate them. As shown in Figure 1d, the training data was randomly split into 5 folds. Four folds were used to train the model and the rest fold was used to estimate the performance of the current hyperparameters in the Random Forest classifier. The performance reported by the 5-fold cross-validation was the average of the values computed in the 5 splits. After the best hyperparameters were determined, the whole 

and the testing data set was used to evaluate the generalizability of the model.

\section{Accuracy evaluation}

250 Accuracy, sensitivity, specificity, precision, and F1-score were calculated to evaluate

251 the performance of the classification (see Figure 1f). In this study, CLBP+ was 252 considered as the positive case and CLBP- was the negative case. Correct predictions 253 of CLBP+ and CLBP- patients are called true positives (TP) and true negatives (TN), 254 respectively. Incorrect classifications of CLBP- patients as CLBP+ or of CLBP+ 255 patients as CLBP-, are called false positives (FP) and false negatives (FN) respectively. 256 Accuracy was the proportion of all the correct classification results.

$$
\text { accuracy }=\frac{T P+T N}{T P+F P+T N+F N}
$$

258 Sensitivity (or recall) represents the proportion of positive cases that are correctly 259 assigned (true positive rate).

$$
\text { sensitivity }=\frac{T P}{T P+F N}
$$

261 Specificity refers to the rate of correctly predicted negative cases in all negative cases 262 (true negative rate).

$$
\text { specificity }=\frac{T N}{T N+F P}
$$

264 Precision is the ratio of the correctly predicted positive cases in all predicted positive cases.

$$
\text { precision }=\frac{T P}{T P+F P}
$$

267 F1-score is the harmonic mean (average) of the precision and sensitivity. 


$$
F 1=\frac{2 \times \text { precision } \times \text { sensitivity }}{\text { precision }+ \text { sensitivity }}
$$

271 The receiver operating characteristic (ROC) curve was calculated to evaluate the 272 performance of the Random Forest classifier. The Y-axis of this curve represents the 273 true positive rate (sensitivity) and the $\mathrm{X}$-axis means false positive rate (1-specificity). 274 Therefore, the overall classification performance of the Random Forest classifier was 275 evaluated by the area under the ROC curve (AUC). A classification model with a larger 276 AUC value has a higher correct rate, and AUC $=1$ means perfect performance. Since 277 the random forest can also output the percentage of trees that vote CLBP+, AUC 278 represents the probability that the random forest outputs a lower percentage for a negative sample than for a positive sample.

\section{Feature importance}

282 SHapley Additive exPlanations (SHAP) [31] was used to explain the gait features 283 importance to the classification model. SHAP connects optimal credit allocation with 284 local explanations using the classic Shapley values from game theory [32]. Shapley 285 values, $\emptyset_{i}$, explains the importance of gait outcome $i$ for Random Forest classifier 286 and is defined as:

$$
\emptyset_{i}=\frac{1}{|N| !} \sum_{\{i\} \in s \text { and } s \subseteq N}(|s|-1) !(|N|-|s|) ![R(s)-R(s-\{i\})]
$$
$N$, and $R()$ is the Random Forest classifier accuracy of the input gait outcomes. Since 
291 a tree explainer to exploit the information stored in the tree structure to calculate the 292 SHAP values which are highly approximate Shapley values. Therefore, higher SHAP 293 values represent higher impact to classify CLBP- and CLBP+ groups.

\section{Results}

295 Demographic characteristics are provided in Table 3. Out of a total of 60 patients, 11 296 were excluded because essential parts of their dataset were incomplete (CSI scores 297 or/and accelerometry data), 7 were excluded since they had less than 4 segments data 298 ( 3 had 1 segment, 2 had 2 segments, and 2 had 3 segments). Therefore, 42 patients were 299 included for the data analysis. Differences between CLBP+ and CLBP- group 300 characteristics (Table 3$)$ were not statistically significant $(p>0.05)$, with exception of 301 CSI score $(\mathrm{p}<0.0001)$ and BSI $(\mathrm{p}=0.01)$.

303 Testing data was used to evaluate the generalizability of the Random Forest classifier 304 and the confusion matrix is shown in in Figure 3. From the confusion matrix, accuracy, 305 sensitivity, specificity, precision, and the F1-score were calculated to evaluate the 306 performance metric of the model. The Random Forest classifier achieved an accurate 307 classification-result (84.4\% accuracy) and the sensitivity and specificity were $75.0 \%$ 308 and $93 \%$ respectively. The precision is $92 \%$ and the F1-score is $82.6 \%$. The ROC curve 309 is presented in Figure 4 showing that the Random Forest classifier achieved a 0.83 AUC. 
Table 3 Patient characteristics $(n=42)$.

\begin{tabular}{lccc}
\hline & CLBP- $(\mathrm{n}=23)$ & CLBP+ $(\mathrm{n}=19)$ & All $(\mathrm{n}=42)$ \\
\hline Gender & $15 \mathrm{~W} / 8 \mathrm{M}$ & $12 \mathrm{~W} / 7 \mathrm{M}$ & $27 \mathrm{~W} / 15 \mathrm{M}$ \\
Age, years & $40.8 \pm 12.8$ & $38.1 \pm 12.7$ & $39.6 \pm 12.6$ \\
Height, cm & $173.5 \pm 10.6$ & $175.7 \pm 8.8$ & $174.5 \pm 9.8$ \\
Weight, kg & $87 \pm 17.7$ & $85.4 \pm 15.1$ & $86.3 \pm 16.4$ \\
Body mass index, kg/m ${ }^{2}$ & $28.9 \pm 5.3$ & $27.7 \pm 4.4$ & $28.3 \pm 4.9$ \\
Central Sensitization Inventory ( 0-100) & $31 \pm 4.8$ & $48.7 \pm 8.7$ & $39.0 \pm 11.2$ \\
Time since pain onset (years) & $4.5 \pm 6.1$ & $3.5 \pm 3.1$ & $4.1 \pm 4.9$ \\
Educational Level & $17 \mathrm{~S} / 6 \mathrm{H}$ & $10 \mathrm{~S} / 9 \mathrm{H}$ & $26 \mathrm{~S} / 15 \mathrm{H}$ \\
Physical demands at work (DOT; Se/Li/Me/He) & $3 / 11 / 8 / 1$ & $4 / 7 / 7 / 1$ & $7 / 18 / 15 / 2$ \\
Patient-reported Pain Intensity (VAS, 0-10) & $5.5 \pm 2$ & $5.2 \pm 1.8$ & $5.4 \pm 1.9$ \\
Disability (PDI, 0-70) & $33.6 \pm 11.2$ & $26.8 \pm 11.9$ & $31.0 \pm 11.7$ \\
Work Ability (WAS, 0-10) & $4.5 \pm 2.3$ & $4.9 \pm 2.8$ & $4.6 \pm 2.5$ \\
Physical Functioning (Rand36-PF, 0-100) & $49.8 \pm 22.3$ & $63.3 \pm 16.1$ & $54.7 \pm 21.1$ \\
Catastrophizing (PCS, 0-52) & $16.3 \pm 8.9$ & $20.3 \pm 11.1$ & $18.1 \pm 10$ \\
Injustice (IEQ, 0-48) & $15.2 \pm 8.9$ & $18.5 \pm 8.5$ & $16.7 \pm 8.8$ \\
Psychological traits Screening (BSI, t-score) & $34.4 \pm 4.9$ & $41.5 \pm 5.8$ & $37.6 \pm 6.4$ \\
\hline
\end{tabular}




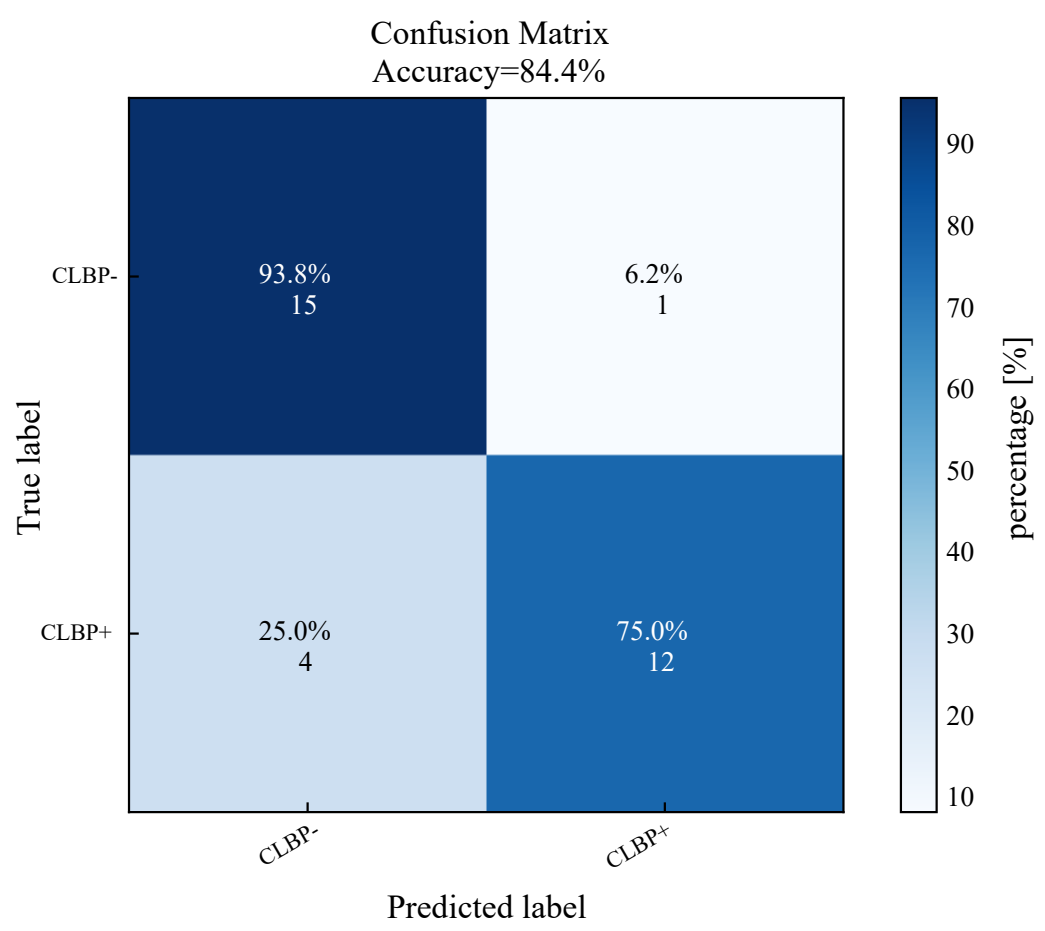

317 Figure 3. Classification results for random forest, and the mean accuracy is $84.4 \%$.

318 CLBP-, CLBP+: Patients with chronic low back pain with low (-) and high (+) central 319 sensitization levels.

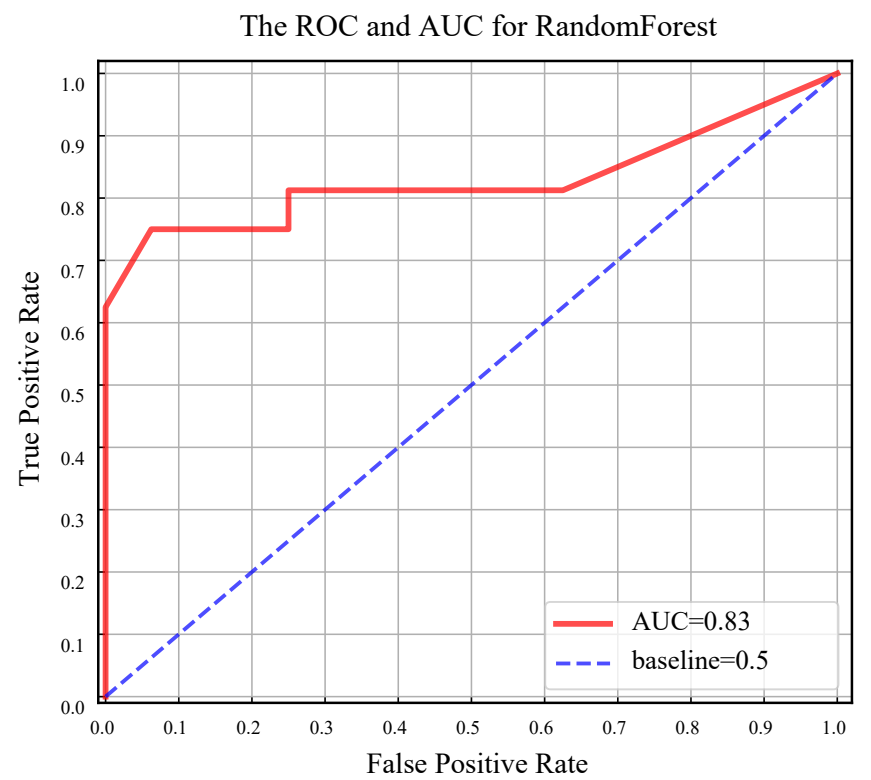

Figure 4. The receiver operating characteristic (ROC) curve (in red) for Random

322 Forest classifier. AUC: arear under the curve. 
Feature Importance

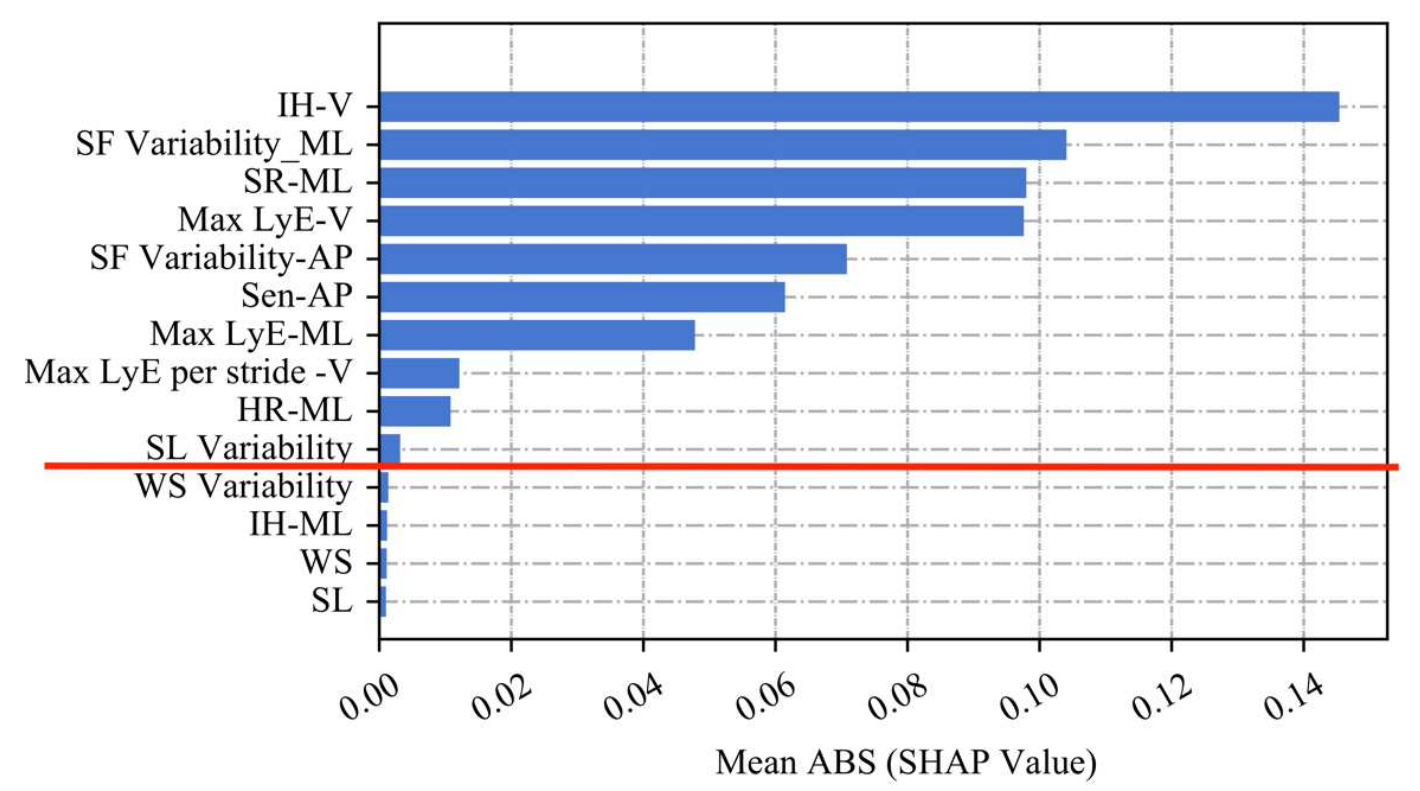

329 Figure 5. Features importance of Random Forest classifier. Top 10 gait outcomes above

330 the red are: index of harmonicity in vertical direction $(\mathrm{IH}-\mathrm{V}), \quad$ variability of stride

331 frequency in mediolateral/anteroposterior direction (SF variability-ML/AP), stride 332 regularity in mediolateral direction (SR-ML), Maximal Lyapunov exponent in 333 vertical/mediolateral direction (Max LyE-V/ML), sample entropy in anteroposterior 334 direction (Sen-AP), Max LyE-V: Maximal Lyapunov exponent per stride in vertical 335 direction, harmonic ration in mediolateral direction (HR-ML) and variability of stride 336 length (SL variability). The rest gait outcomes below the red line are: WS variability: 337 variability of walking speed, IH-ML: index of harmonicity in mediolateral direction, 
WS: mean walking speed and SL: mean stride length. ABS: absolute value. SHAP:

SHapley Additive exPlanations.

Figure 6 shows the violin-box plot of the Top 10 important gait outcomes. Violin-box plot is a hybrid of a kernel density plot and a box plot, and the dots show the individuals data. A box plot contains a set of whiskers, a box and a horizontal line in the middle of the box, representing the minimum, maximum, first quartile, third quartile and median of the data respectively. From this figure, it is easy to distinguish the differences of the median between each gait outcomes. It shows that CLBP- group has higher IH-V, HRML (better smoothness); higher SF-variance-ML, SF-variance-AP. SL-variance (lesser variability); lower SR-ML (lesser regularity), lower Max LyE-V, Max LyE-per-strideV, slightly lower Max LyE-ML (better stability); and slightly higher Sen-AP (lesser predictability). Although the differences of medians between 2 groups in Sen-AP and

351 Max LyE-ML are small, their distributions are different. In Sen-AP, data of CLBP- has 352 a wider distribution and CLBP+ shows more data at the bottom. In the Max LyE-ML, 353 data of CLBP- is concentrated on median while CLBP+ has a wide distribution and a 354 lower peak. For other gait outcomes, the distributions are also different. In IH-V, 355 distributions of CLBP- and CLBP+ all showed a bimodality distribution but the peaks 356 of distribution are totally different. In SF Variability-ML and SF Variability-AP, 357 CLBP+ has a larger peak at the bottom while CLBP- has a wide range distribution. 358 Similarly, in SR-ML, CLBP+ has a concentrating distribution while the peak of CLBP359 is lower. In Max LyE-V and Max LyE per stride -V, CLBP- shows a log-normal 
distribution while CLBP+ shows a wider distribution. In HR-ML and SL Variability,

361 the distributions are similar but CLBP+ has more outliers.
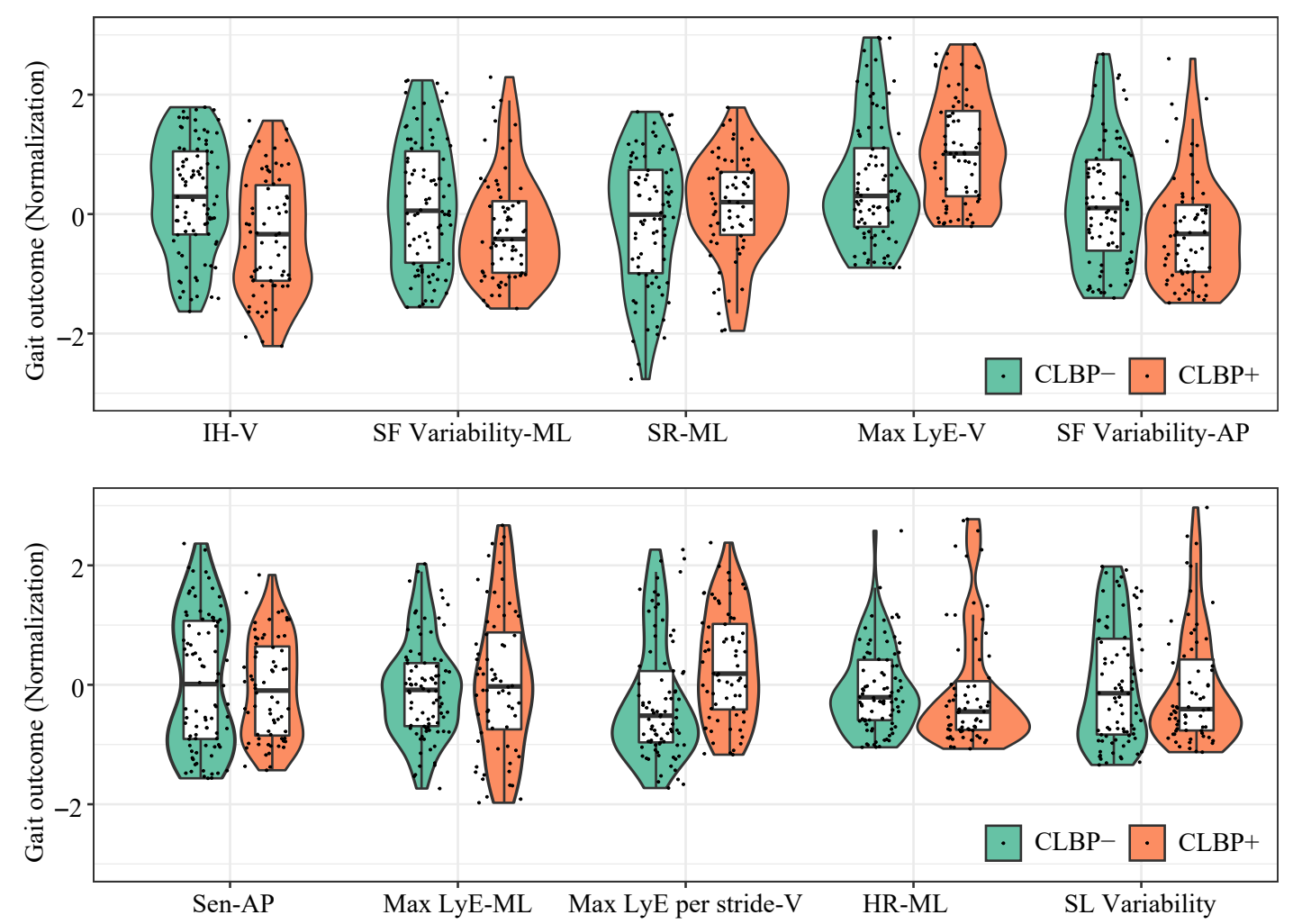

364 Figure 6. Violin-box plot for the top 10 gait outcomes. Dots show the individuals data.

CLBP-, CLBP+: Patients with chronic low back pain with low (-) and high (+) CS levels. IH-V: index of harmonicity in vertical direction, SF variability-ML/AP: variability of stride frequency in mediolateral/ anteroposterior direction, SR-ML: stride regularity in mediolateral direction, Max LyE-V/ML: Maximal Lyapunov exponent in vertical/mediolateral direction, Sen-AP: sample entropy in anteroposterior direction and HR-ML: harmonic ration in mediolateral direction. 


\section{Discussion}

374 The aim of this study was to analyze whether and how the presence of CS is related to 375 differences in gait performance of patients with CLBP during daily life by using a 376 machine learning method. Based on quantitative and qualitative gait outcomes, using a 377 Random Forest method, the two groups (CLBP- and CLBP+) could be classified with 378 an accuracy of $84.4 \%$. The classification results indicated that CLBP- patients walk 379 differently from CLBP+. Furthermore, the SHAP values showed that the differences 380 between CLBP- and CLBP+ groups were present in gait outcomes that represented 381 smoothness, stability, predictability, regularity and variability.

383 In the present study, we addressed the walking of patients with CLBP in a daily-living

384 environment. Walking in a controlled laboratory or during a clinical assessment is 385 different from self-initiated gait, during activities of daily living. Walking in daily life, 386 might be subject to environmental perturbations, quick changes while performing a task, 387 and often involves the performance of several actions at the same time [33], e.g. 388 walking when carrying a cup of coffee. These influences on gait are not present in 389 controlled studies, and are not captured by averaged based conventional gait outcomes 390 that average outcomes over stride cycles, such as mean, step length, stride or step time, 391 and number of steps. Therefore, in the present study we included gait outcomes that 392 take into account how gait cycles evolve over time, e.g. the interdependency of gait 393 cycles, using sample entropy as a measure of predictability of the gait pattern, the 
maximal Lyapunov exponent as quantification of local stability and correlation-based measures that take into account how gait cycles evolve over time [34].

The Random Forest method differs from conventional statistical methods, such as the T-test which does not consider the interaction of gait outcomes and simply evaluates the differences of each gait outcome one by one. The Random Forest method is an ensemble of decision trees and incorporating gait outcomes interactions naturally in the 401 classification process. For example, a decision tree with depth 2 from the Random 402 Forest, with the father node IH-V and the son node Sen-AP, can describe an interactive 403 gait pattern: if IH-V >* and Sen-AP $>^{*}$, the data belong to CLBP-. Since the Random 404 Forest includes multiple decision trees and each tree is built based on a random subset 405 of gait outcomes, Random Forest can capture the complex interaction of gait outcomes. 406 Additionally, it can help to reduce the chance of overfitting to training data. Therefore, 407 the Random Forest improves predictive accuracy and it can provide a generalized 408 model of the difference between CLBP- and CLBP+ groups.

410 The SHAP tree explainer can provide good explanations for the Random Forest [31]. It

411 fastens the calculation of the SHAP values by exploiting the information stored in the 412 tree structure which already captured the gait outcomes interactions. The top 10 SHAP 413 values suggest that the differences between CLBP- and CLBP+ groups are gait 414 outcomes which represent smoothness, stability, predictability, regularity and 415 variability in gait. Compared with CLBP- group, CLBP+ group exhibited worse 
smoothness and local stability in gait. Moreover, the CLBP+ group exhibited a more regular, less variable and more predictable gait pattern.

Most studies on walking in patients with CLBP are compared with control participants with no back pain. To the best of our knowledge, this is the first study in patients with

CLBP that addresses the difference in gait pattern between two CLBP groups based on low and high CS level, which makes a direct comparison with other studies intricate. However, the results of different gait patterns between low and high CS level support the notion that within the heterogenous CLBP group, different motor control strategies are adopted. Two motor control strategies on a continuum have been suggested with

There is considerable evidence on the observed changes in muscle activation of patients with CLBP [36]. Tight control which involves increased trunk muscle activation and

431 enhanced muscle co-contraction, might enhance control over trunk posture and 432 movement [35]. Increased muscle activation and enhanced co-contraction would help 433 individuals to maintain the stability of lumbar spine [37], which is an unstable structure.

434 However, this strategy might impair patients' ability to maintain balance in a complex daily-living environment where unstable surfaces and environmental perturbations occur [38]. Increased co-contraction would reduce the demand for the intricate control 437 of the sequences of muscle activation. It might avoid the potential error raised by 
inaccurate sensory feedback of CLBP [35]. This might allow patients to control their trunks' movement precisely and result in lower variability of movement patterns [39]. Our results might infer that CLBP+ group exhibited a more "tight" control. Tight control allows them to control their movement more strictly with results in a decreased motor variability, higher regularity and predictability of the gait pattern compared with CLBP-group.

The CLBP- group on the other hand might use another 'loose control' strategy. The "Loose control", which involves reduced muscle excitability, might reduce the control over trunk movements [35]. The spine of which each spinal unit has 6 degrees of freedom, is controlled by its surrounding musculature. Reduced muscular excitability, leads to a reduced control over the spinal muscle, to larger amplitude movements, and to more movement variability during repeated tasks [35]. Increased motor variability might probably prevent muscle fatigue [40] since it allows sharing the load between different structures or tissues. Moreover, motor variability makes it possible to explore new pain-free motor control solutions [41]. The results of the present study hint at a more 'loose control' in the CLBP- group, which increased motor variability, which might allow them to flexibly adapt to the complex daily-living environment using different movement solutions. Additionally, irrespective of the larger variability local stability and smoothness of the gait pattern was higher in this group than in the CLBP+ group. 
Although both motor control adapted strategies might have beneficial effects for the

461 short term, they might lead to negative long-term consequences. Increased muscle

462 activation and co-contraction in tight control would increase the forces acting on the 463 spine and it would lead to higher spinal loading. Moreover, even when patients are at 464 rest, the co-contraction of muscles is continuous [42]. These might result in 465 accumulation of waste products, muscle fatigue, and intervertebral disc degeneration 466 [40][43]. Regarding loose control, the reduced control of lumbar spine may eventually 467 increase the tissue strains, with subsequent increases in spinal loading, and pain [41]. 468 Thus, eventually both strategies affect mechanical loading on lumbar tissues. The 469 loading might be the source of nociceptive input and might contribute to the CS since 470 the load may sufficient to excite sensitized afferents [35].

472 Clinically, the important gait outcomes of this study may assist clinicians in providing 473 a more accurate understanding of the gait performance of patients with CLBP, with low 474 or high CS levels, and a more explicit operationalization of the observed "abnormal" 475 gait pattern of patients with chronic pain. Whether "abnormal" should be interpreted as 476 a functional or a dysfunctional motor control strategy in the short or long term, remains 477 to be studied. The approaches used in this study have presented a novel way to identify 478 interacting feature, and therefore, can be used for further studies. Clinically, the present 479 accurate subclassification could become meaningful if this would lead to effective 480 treatment approaches. While this cross-sectional study has objectified a relation 481 between CS and gait features, the direction of this relation is unknown. Follow-up 
studies would benefit from a longitudinal design with multiple measurements to help further unraveling of this relation, as well as the relation to disability.

In line with most studies on walking and CLBP, we used cross-sectional data, thus we are not allowed to infer causality between motor control changes, CS and CLBP. Moreover, we labeled the groups based on CSI score and the cut off values from a previous study [25]. It should also be noted that a gold standard measure to diagnose CS is unavailable. The CSI is regarded as an indirect measure of CS, because higher scores are associated with the presence of CS syndromes [25]. In addition to gait assessment, it would be interesting to explore differences in physical activities between CLBP- and CLBP+, because several studies reported that relationship between CLBP and physical activity levels is heterogeneous [44].

\section{Conclusion}

The present study analyzed gait data during daily living of CLBP patients with low and high CS levels. A Random Forest method and the SHAP method were applied for classification and identification the contribution of gait outcomes to the model. This analytic approach demonstrated that Random Forest method has the ability to accurately classify subgroups of patients with CLBP and low or high CS levels based on differences in gait outcomes. The results of SHAP method showed the differences between low and high CS levels were in gait regularity, variability, predictability, smoothness and stability. The differences in gait outcomes may infer that patients with 
504 low and high CS levels adopted different motor control strategies. Patients with CLBP

505 and low CS level (CLBP-) may use a more loose control and, therefore, exhibited more

506 smoothness and stability in gait patterns. Patients with CLBP and high CS level

$507(\mathrm{CLBP}+)$ may adopt a more tight control and showed a more regular, less variable and 508 more predictable gait pattern.

509

510 The results of this study may contribute to a better understanding of gait characteristics

511 in patients with CLBP, its association with CS, and may in the future assist in better-

512 personalized rehabilitation interventions [45].

513

514 List of abbreviations

515 CLBP: chronic low back pain

516 CS: central sensitization

517 CSI: Central Sensitization Inventory

518 CLBP-: chronic low back pain with low central sensitization

519 CLBP+: chronic low back pain with high central sensitization

$520 \quad$ V: vertical direction

521 ML: mediolateral direction

522 AP: anteroposterior direction

523 WS: walking speed

524 SL: Stride length

525 ST: Stride time 
527 RMS: Root mean square of the variability of the amplitude of accelerations

528 SR: Stride regularity

529 GSI: Gait symmetry index

$530 \quad$ IH: Index of harmonicity

531 HR: Harmonic ratio

532 Sen: Sample entropy

533 max LyE: Maximal Lyapunov exponent

534 max LyE per stride: Maximal Lyapunov exponent normalized per stride by time

535 TP: true positives

536 TN: true negative

537 FP: false positives

538 FN: false negative

539 ROC: receiver operating characteristic

540 AUC: area under the receiver operating characteristic curve

541 SHAP: SHapley Additive exPlanations

\section{Declarations}

\section{Ethics approval and consent to participate}

544 The study was approved by the Medical Research Ethics Committee of the University

545 Medical Center Groningen (METc 2016/702) and conducted according to the principles

546 expressed in the Declaration of Helsinki. 
549 Not applicable.

550

$551 \quad$ Availability of data and materials

552 Not applicable.

553

554 Competing interests

555 The authors declare that they have no competing interests.

556

557 Funding

558 XZ was supported by China Scholarship Council-University of Groningen

559 Scholarship under Grant No.201906410084.

560

561 Authors' contributions

562 XZ, MR, EO and CL developed the idea. JAE and HRSP collected the data from 563 participants. XZ analyzed the data and wrote the paper under supervision of MR, OB 564 and CL. HK reviewed the code. All authors reviewed and commented on the manuscript. 565 All authors approved the final manuscript.

\section{Acknowledgements}

568 Not applicable. 


\section{$571 \quad$ Reference}

572 [1] Thiese, MS, Hegmann KT, Wood EM, Garg A, Moore JS, Kapellusch J, et al. Prevalence of 573 low back pain by anatomic location and intensity in an occupational population. BMC $574 \quad$ musculoskeletal disorders, 2014;15:283.

575 [2] Dagenais S, Caro J, Haldeman S. A systematic review of low back pain cost of illness studies in the United States and internationally. The spine journal. 2008;8:8-20.

577 [3] Hartvigsen J, Hancock MJ, Kongsted A, Louw Q, Ferreira ML, Genevay S, et al. What low 578 back pain is and why we need to pay attention. The Lancet. 2018;391:2356-2367.

579 [4] Airaksinen O, Brox JI, Cedraschi C, Hildebrandt J, Klaber-Moffett J, Kovacs F, et al. European 580 guidelines for the management of chronic nonspecific low back pain. European spine journal. 2006;15 Suppl 2: s192.

582 [5] Nijs J, Van Wilgen CP, Van Oosterwijck J, van Ittersum M, Meeus M. How to explain central sensitization to patients with 'unexplained'chronic musculoskeletal pain: practice guidelines. Manual therapy. 2011;16:413-418.

[6] van Tulder MW, Koes BW, Bouter LM. A cost-of-illness study of back pain in The Netherlands. Pain. 1995;62:233-240.

[7] Jenkinson RH. Central Sensitization. Top Pain. 2019. doi:10.1007/978-3-319-99124-5_11.

588 [8] Foster N E, Anema J R, Cherkin D, Chou R, Cohen SP, Gross DP, et al. Prevention and 589 treatment of low back pain: evidence, challenges, and promising directions. The Lancet. 2018;391:2368-2383.

591 [9] Maher C, Underwood M, Buchbinder R. Non-specific low back pain. The Lancet. 
593 [10] Götze M, Ernst M, Koch M, Blickhan R. Influence of chronic back pain on kinematic reactions to unpredictable arm pulls. Clinical Biomechanics. 2015,30:290-295.

595 [11] Giesecke T, Gracely RH, Grant MAB, Nachemson A, Petzke F, Williams DA, et al. Evidence 596 of augmented central pain processing in idiopathic chronic low back pain. Arthritis \& Rheumatism: Official Journal of the American College of Rheumatology. 2004;50:613-623.

[12] Ahern DK, Follick MJ, Council JR, Laser-Wolston N, Litchman H. Comparison of lumbar paravertebral EMG patterns in chronic low back pain patients and non-patient controls. Pain.

601 [13] Lamoth CJC, Meijer OG, Daffertshofer A, Wuisman PI, Beek PJ. Effects of chronic low back 602

[14] Lamoth CJC, Stins JF, Pont M, Kerckhoff F, Beek PJ. Effects of attention on the control of locomotion in individuals with chronic low back pain. Journal of Neuroengineering and Rehabilitation. 2008;5:1-8.

[15] Hamacher D, Hamacher D, Herold F, Schega L. Are there differences in the dual-task walking variability of minimum toe clearance in chronic low back pain patients and healthy controls? Gait \& Posture. 2016; 49:97-101.

[16] Christe G, Kade F, Jolles BM, Favre J. Chronic low back pain patients walk with locally altered spinal kinematics. Journal of Biomechanics. 2017;60:211-218.

612 [17] Gombatto SP, Brock T, DeLork A, Jones G, Madden E, Rinere C. Lumbar spine kinematics 613 during walking in people with and people without low back pain. Gait \& Posture. 2015;42:539- 
615 [18] Müller R, Ertelt T, Blickhan R. Low back pain affects trunk as well as lower limb movements 616 during walking and running. Journal of Biomechanics. 2015;48:1009-1014.

617 [19] Echeita JA, Preuper HRS, Dekker R, Stuive I, Timmerman H, Wolff AP, et al. Central 618 Sensitisation and functioning in patients with chronic low back pain: protocol for a crosssectional and cohort study. BMJ Open. 2020;10:e031592.

620 [20] Zhou Y, Romijnders R, Hansen C, van Campen J, Maetzler W, Hortobágyi T, et al. The 621 detection of age groups by dynamic gait outcomes using machine learning approaches. Scientific Reports. 2020;10:1-12.

623 [21] van Schooten KS, Pijnappels M, Rispens SM, Elders PJ, Lips P, van Dieen JH. Ambulatory fall-risk assessment: amount and quality of daily-life gait predict falls in older adults. Journals

[22] Del Din S, Godfrey A, Galna B, Lord S, Rochester L. Daily-living gait characteristics in ageing and Parkinson's disease: impact of environment and ambulatory bout length. Journal of

[23] Vickers J, Reed A, Decker R, Conrad BP, Olegario-Nebel M, Vincent HK. Effect of Neuroengineering and Rehabilitation. 2016;13:46. investigator observation on gait parameters in individuals with and without chronic low back

632 [24] van Wilgen CP, Meeus M, Descheemaeker F, Cagnie B. Central Sensitization Inventory 633 Nederlandse consensusvertaling. 2013. Available: http://www.paininmotion.be/storage/app/media//materials/CSI-consensusvertaling.pdf. 
[25] Neblett R, Cohen H, Choi Y H, Hartzell MM, Williams M, Mayer TG, \& Gatchel RJ. The Central Sensitization Inventory (CSI): establishing clinically significant values for identifying central sensitivity syndromes in an outpatient chronic pain sample. The Journal of Pain.

$$
2013 ; 14: 438-445 \text {. }
$$

[26] Ichinoseki-Sekine N, Kuwae Y, Higashi Y, Fujimoto T, Sekine M, Tamura T. Improving the accuracy of pedometer used by the elderly with the FFT algorithm. Medicine \& Science in Sports \& Exercise. 2006; 38:1674-1681.

[27] Qian J, Pei L, Ma J, Ying R, Liu P. Vector graph assisted pedestrian dead reckoning using an unconstrained smartphone. Sensors. 2015;15:5032-5057.

[28] Van Schooten KS, Pijnappels M, Rispens SM, Elders PJ, Lips P, Daffertshofer A, et al. Dailylife gait quality as predictor of falls in older people: a 1-year prospective cohort study. PLoS One. 2016;11: e0158623.

[29] Kikkert LHJ, Vuillerme N, van Campen JP, Appels BA, Hortobágyi T, \& Lamoth CJC. Gait characteristics and their discriminative power in geriatric patients with and without cognitive impairment. Journal of neuroengineering and rehabilitation. 2017;14:1-10.

[30] Liaw A, Wiener M. Classification and regression by randomForest. R News. 2002;2:18-22.

[31] Lundberg SM, Lee SI. A unified approach to interpreting model predictions. Advances In Neural Information Processing Systems. 2017:4765-4774.

[32] Shapley LS. A value for n-person games. Contributions to the Theory of Games. 1953;2:307317.

[33] Hillel I, Gazit E, Nieuwboer A, Avanzino L, Rochester L, Cereatti A, \& et al. Is every-day walking in older adults more analogous to dual-task walking or to usual walking? Elucidating 

aging and physical activity. 2019;16:1-12.

[34] Lamoth CJC, Ainsworth E, Polomski W, Houdijk H. Variability and stability analysis of walking of transfemoral amputees. Medical Engineering \& Physics. 2010;32:1009-1014.

[35] Van Dieën J H, Reeves N P, Kawchuk G, Van Dillen LR, \& Hodges PW. Motor control changes in low back pain: divergence in presentations and mechanisms. Journal of Orthopaedic \& Sports Physical Therapy. 2019;49:370-379.

[36] Prins MR, Griffioen M, Veeger TT, Kiers H, Meijer OG, van der Wurff P, et al. Evidence of splinting in low back pain? A systematic review of perturbation studies. European Spine Journal. 2018;27:40-59.

[37] Cholewicki J, Simons AP, Radebold A. Effects of external trunk loads on lumbar spine stability. Journal of biomechanics. 2000;33:1377-85.

[38] Mok NW, Hodges PW. Movement of the lumbar spine is critical for maintenance of postural recovery following support surface perturbation. Experimental brain research. 2013;231:30513.

[39] Van den Hoorn W, Bruijn SM, Meijer OG, Hodges PW \& Van Dieën JH. Mechanical coupling between transverse plane pelvis and thorax rotations during gait is higher in people with low back pain. Journal of biomechanics. 2012;45,342-347.

[40] Madeleine P. On functional motor adaptations: from the quantification of motor strategies to the prevention of musculoskeletal disorders in the neck-shoulder region. Acta Physiologica. 2010;199:1-46.

[41] Meier ML, Vrana A, Schweinhardt P. Low back pain: the potential contribution of supraspinal 

motor control and proprioception. The Neuroscientist. 2019;25:583-96.

681 [42] Schinkel IA, Nairn BC, Drake JD. Investigation of trunk muscle co-contraction and its 682 association with low back pain development during prolonged sitting. Journal of 683 Electromyography and Kinesiology. 2013;23:778-86.

684 [43] Paul CP, Schoorl T, Zuiderbaan HA, Doulabi BZ, van der Veen AJ, van de Ven PM, et al. 685 Dynamic and static overloading induce early degenerative processes in caprine lumbar $686 \quad$ intervertebral discs. PloS one. 2013;8:e62411.

687 [44] Parker R, Bergman E, Mntambo A, Stubbs S, \& Wills M. Levels of PA in people with chronic 688 pain. The South African Journal of Physiotherapy. 2017;73:1.

689 [45] Van Dieën J H, Reeves N P, Kawchuk G, Van Dillen LR. Analysis of motor control in patients 690 with low back pain: a key to personalized care?. Journal of Orthopaedic \& Sports Physical 691 Therapy. 2019;49:380-388. 


\section{Figures}

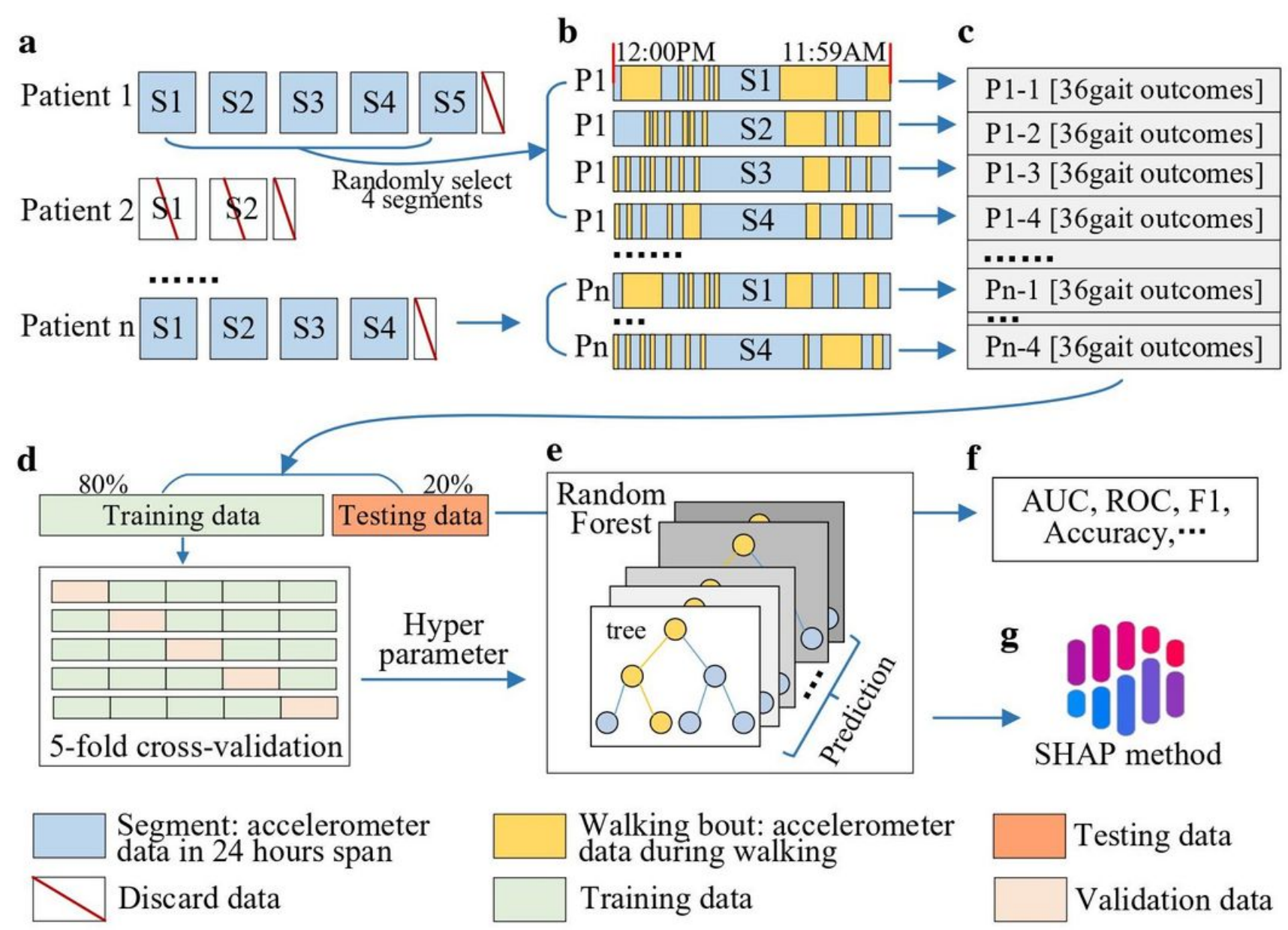

\section{Figure 1}

The data processing and analysis: (a) raw data segmentation, (b) walking bouts extraction, (c) gait outcome vectors, (d) training and testing data preparation, (e) Random Forest classifier, (f) accuracy evaluation, (g) feature importance 


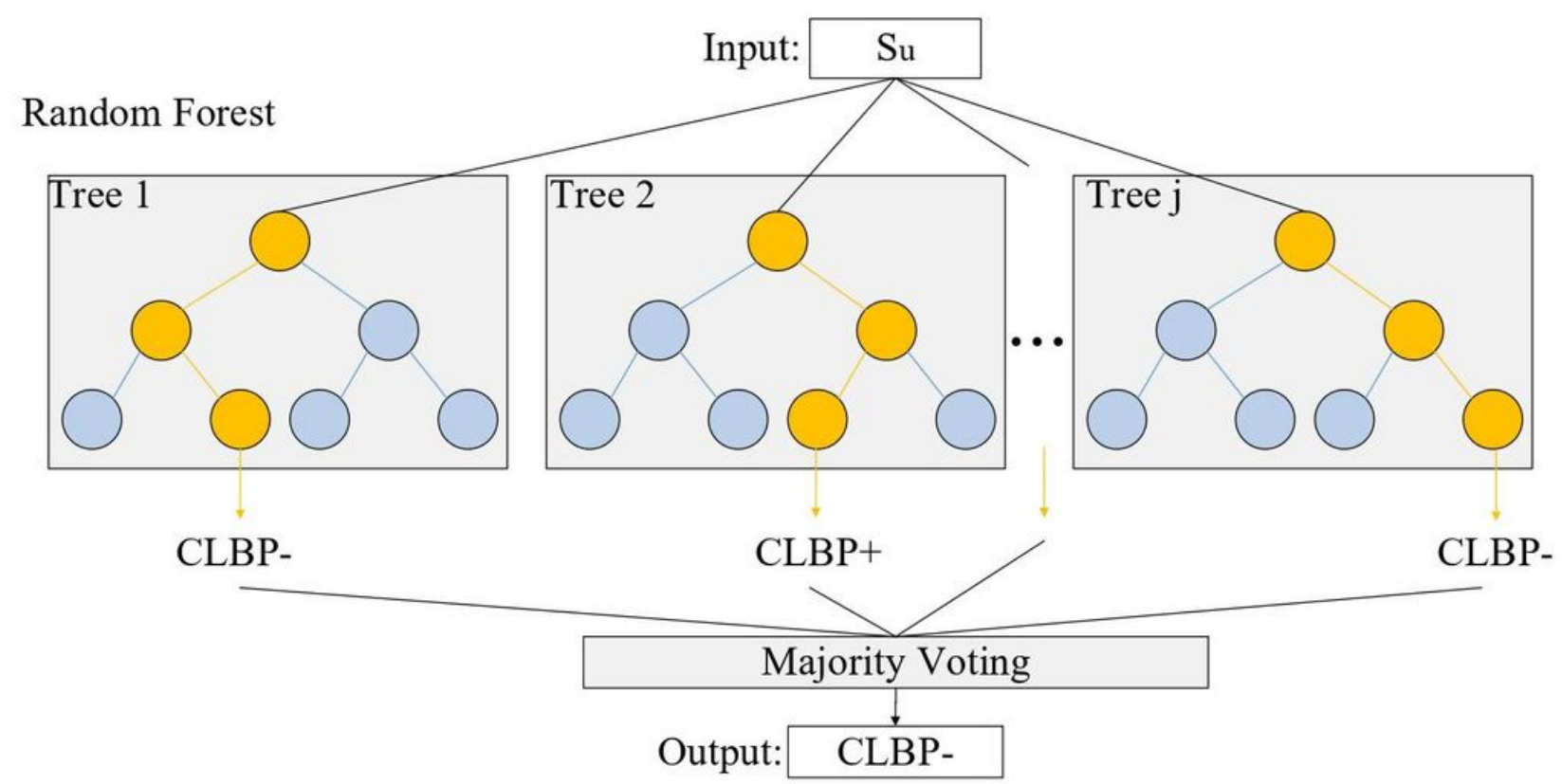

Figure 2

Architecture of random forest. 


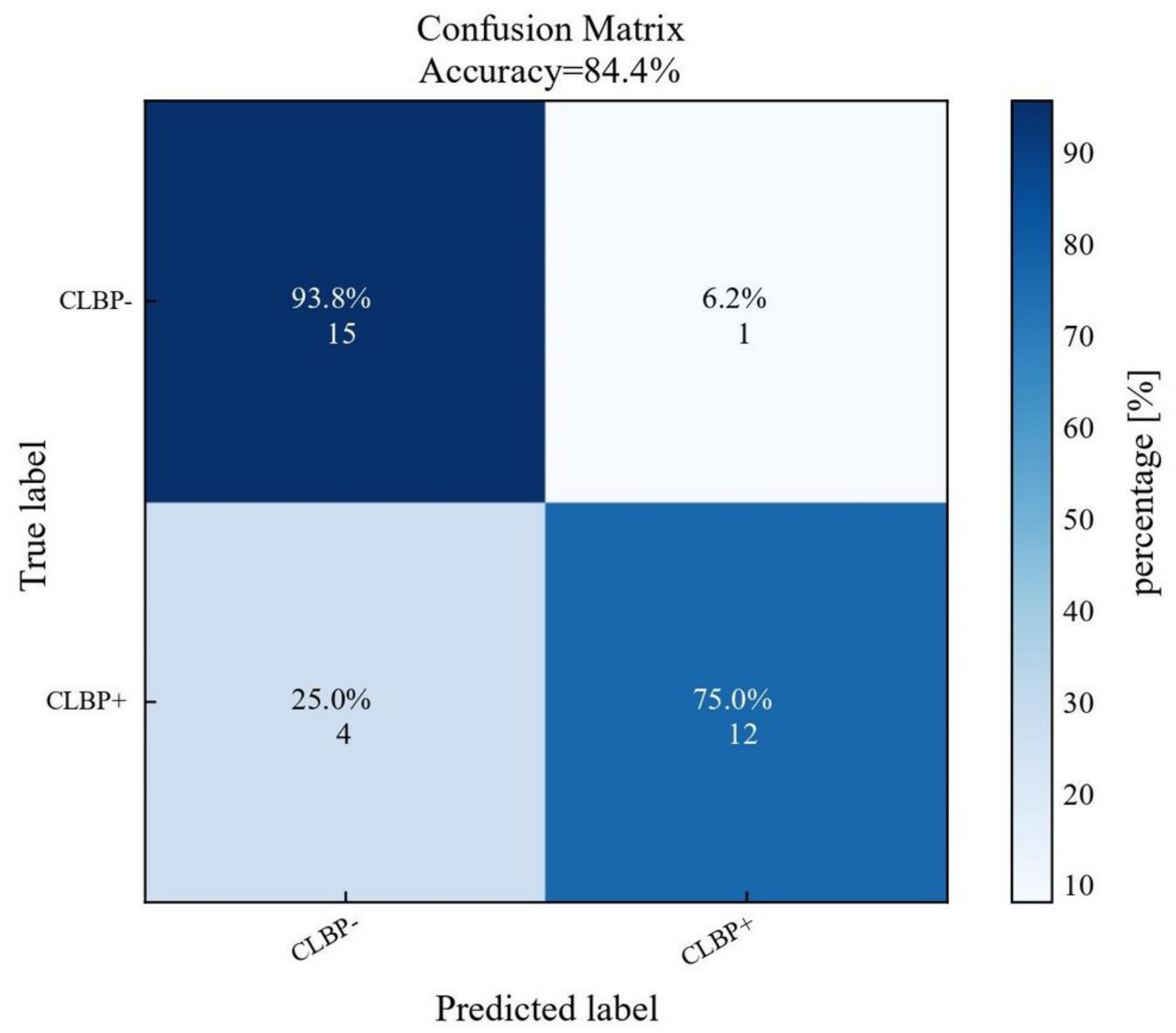

Figure 3

Classification results for random forest, and the mean accuracy is $84.4 \%$. CLBP-, CLBP+: Patients with chronic low back pain with low (-) and high (+) central sensitization levels. 


\section{The ROC and AUC for RandomForest}

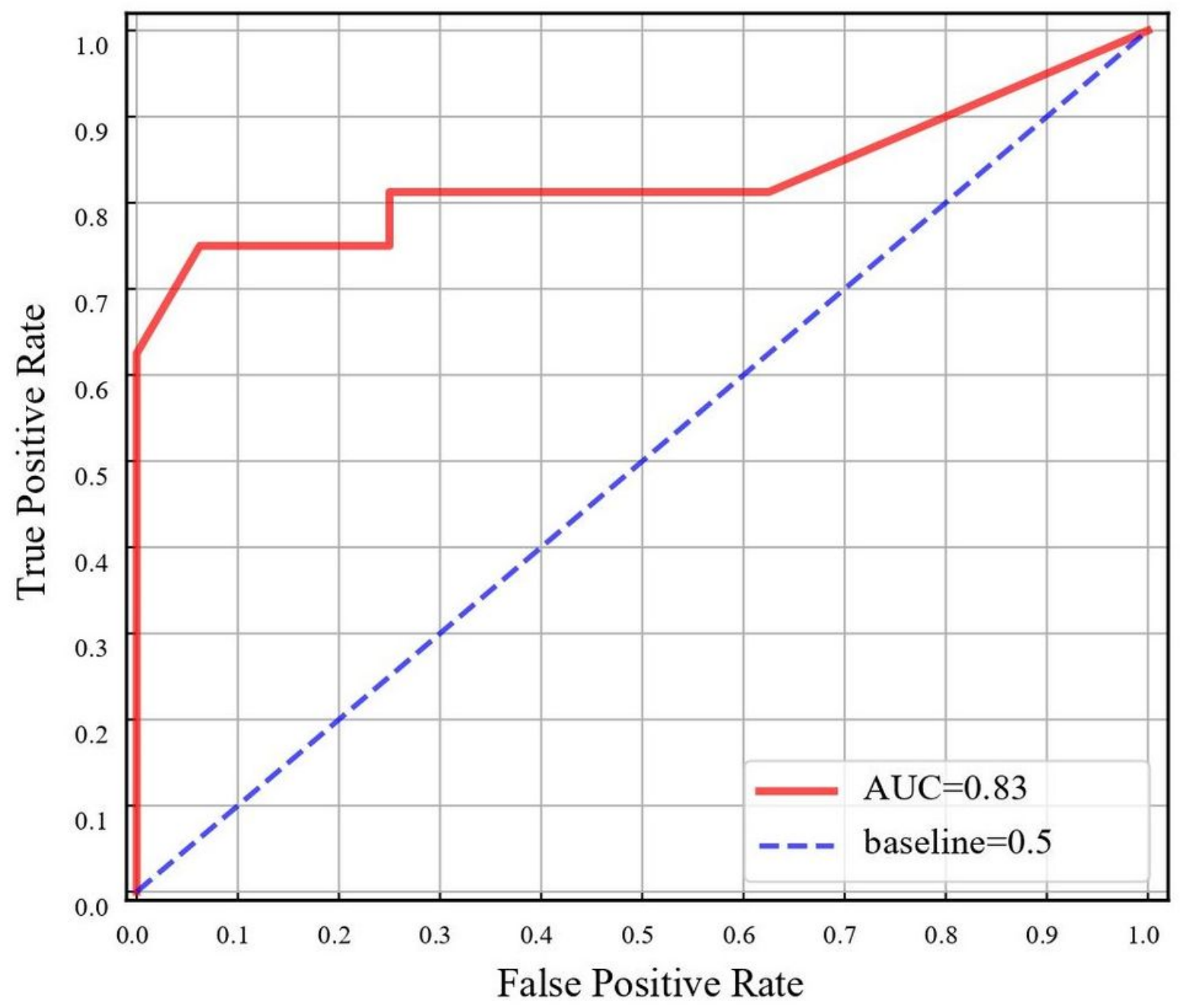

Figure 4

The receiver operating characteristic (ROC) curve (in red) for Random Forest classifier. AUC: arear under the curve. 


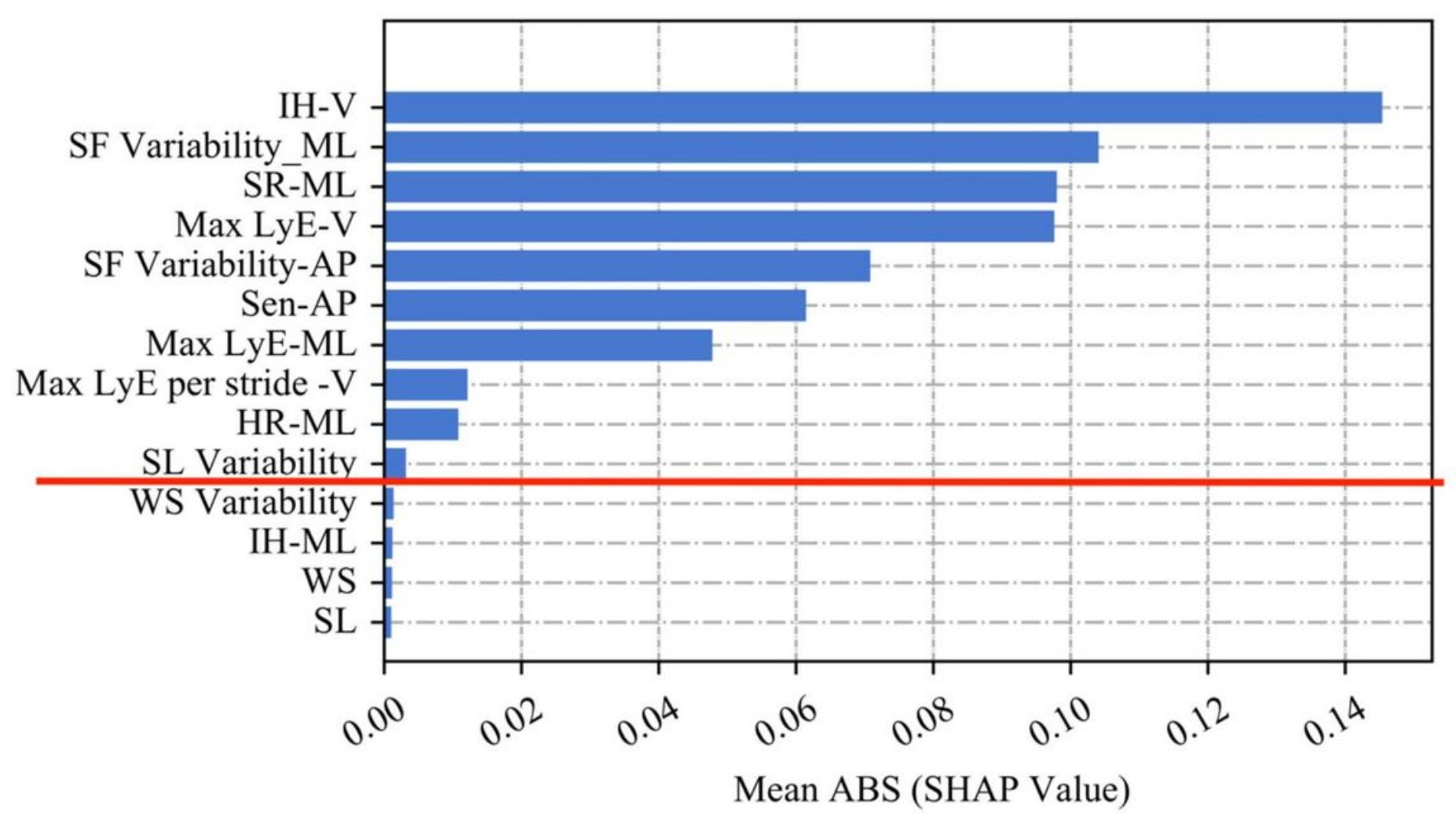

\section{Figure 5}

Features importance of Random Forest classifier. Top 10 gait outcomes above the red are: index of harmonicity in vertical direction $(\mathrm{IH}-\mathrm{V})$, variability of stride frequency in mediolateral/anteroposterior direction (SF variability-ML/AP), stride regularity in mediolateral direction (SR-ML), Maximal Lyapunov exponent in vertical/mediolateral direction (Max LyE-V/ML), sample entropy in anteroposterior direction (Sen-AP), Max LyE-V: Maximal Lyapunov exponent per stride in vertical direction, harmonic ration in mediolateral direction (HR-ML) and variability of stride length (SL variability). The rest gait outcomes below the red line are: WS variability: variability of walking speed, $\mathrm{IH}-\mathrm{ML}$ : index of harmonicity in mediolateral direction, WS: mean walking speed and SL: mean stride length. ABS: absolute value. SHAP: SHapley Additive exPlanations 

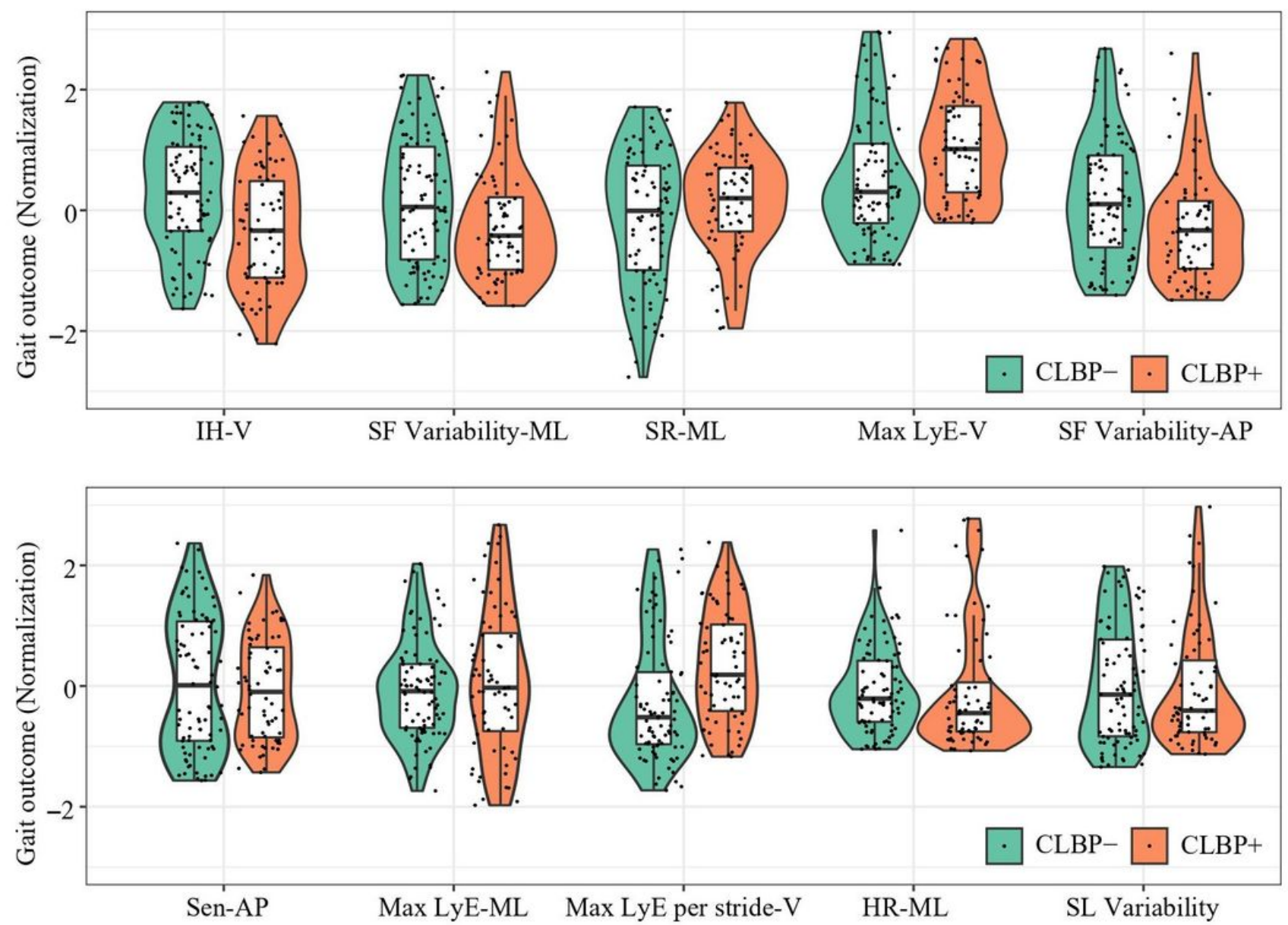

Figure 6

Violin-box plot for the top 10 gait outcomes. Dots show the individuals data. CLBP-, CLBP+: Patients with chronic low back pain with low (-) and high (+) CS levels. IH-V: index of harmonicity in vertical direction, SF variability-ML/AP: variability of stride frequency in mediolateral/ anteroposterior direction, SR-ML: stride regularity in mediolateral direction, Max LyE-V/ML: Maximal Lyapunov exponent in vertical/mediolateral direction, Sen-AP: sample entropy in anteroposterior direction and HR-ML: harmonic ration in mediolateral direction.

\section{Supplementary Files}

This is a list of supplementary files associated with this preprint. Click to download.

- supportinginformation.pdf 